\title{
Birt-Hogg-Dubé syndrome in Korean: clinicora- diologic features and long term follow-up
}

Joo Hee Lee ${ }^{1, *}$, Min Joo Jeon ${ }^{1, *}$, Joon Seon Song ${ }^{2}$, Eun Jin Chae ${ }^{3}$, Jin-Ho Choi ${ }^{4}$, Gu-Hwan Kim , and Jin Woo Song ${ }^{1}$

Departments of ${ }^{1}$ Pulmonary and Critical Care Medicine, ${ }^{2}$ Pathology, ${ }^{3}$ Radiology, ${ }^{4}$ Pediatrics, and ${ }^{5}$ Medical Genetics Center, Asan Medical Center, University of Ulsan College of Medicine, Seoul, Korea

Received: March 31, 2018

Revised : May 7, 2018

Accepted: May 10, 2018

Correspondence to

Jin Woo Song, M.D.

Department of Pulmonary and Critical Care Medicine, Asan

Medical Center, University of

Ulsan College of Medicine, 88

Olympic-ro 43-gil, Songpa-gu,

Seoul 05505, Korea

Tel: +82-2-3010-3993

Fax: +82-2-3010-6968

E-mail:jwsong@amc.seoul.kr

*These authors contributed equally to this work.
Background/Aims: Birt-Hogg-Dubé (BHD) syndrome is a rare autosomal dominant disorder that is characterized by skin fibrofolliculomas, pulmonary cysts, and renal tumors. The objective of this study was to describe the features of Korean patients with BHD syndrome.

Methods: Clinical data were retrospectively reviewed in 12 patients (10 confirmed by direct sequencing of the folliculin (FLCN) gene and two confirmed by clinical diagnosis) diagnosed from 2004 to 2016 at Asan Medical Center, Seoul, South Korea. Criteria proposed by the European BHD consortium were used for diagnosis. Results: The median follow-up was 52 months. The mean age was 41.3 years and $66.7 \%$ were female. Eight patients $(66.7 \%)$ had a history of pneumothorax, which was recurrent in $75 \%$. Skin lesions were detected in $25.0 \%$ and renal cancer in $25.0 \%$. Among mutations of the FLCN gene, the duplication of cytosine in the C8 tract of exon 11 (c.1285dupC) was the most common (40\%); however, a novel heterozygous sequence variant of c.31T>C (p.C11R) in exon 4 was detected in one patient. All patients had multiple and bilateral pulmonary cysts, distributed in predominantly lower, peripheral and subpleural regions of the lungs. Most patients showed preserved lung function that remained unchanged during follow-up, and two (16.7\%) developed cancers (renal cancer in one and breast cancer in one). Conclusions: Our data suggest that Korean patients with BHD syndrome may have a higher risk of pneumothorax, less frequent skin lesions, and a novel FLCN mutation compared to previous reports. Multiple bilateral and basal-predominant cysts were the most common radiologic features.

Keywords: Birt-Hogg-Dube syndrome; FLCN gene; Pneumothorax; Carcinoma, renal cell; Fibrofolliculoma

\section{INTRODUCTION}

Birt-Hogg-Dubé (BHD) syndrome, initially described in 1977 [1], is a rare autosomal dominant disorder (100 to 400 families detected worldwide) that is characterized by the presence of skin fibrofolliculomas, pulmonary cysts, and renal tumors. The syndrome is caused by germline mutations of the folliculin (FLCN) gene located on chro- mosome 17p11.2, which is a tumor suppressor gene that produces folliculin [2]. Folliculin is preferentially expressed in the skin, kidney, and lung and is involved in the mammalian target of rapamycin signaling pathway regulating cell growth and metabolism [3].

Several previous Japanese studies have suggested that Japanese patients with BHD syndrome have less frequent skin lesions than white patients [4-7]. Gunji et al. 


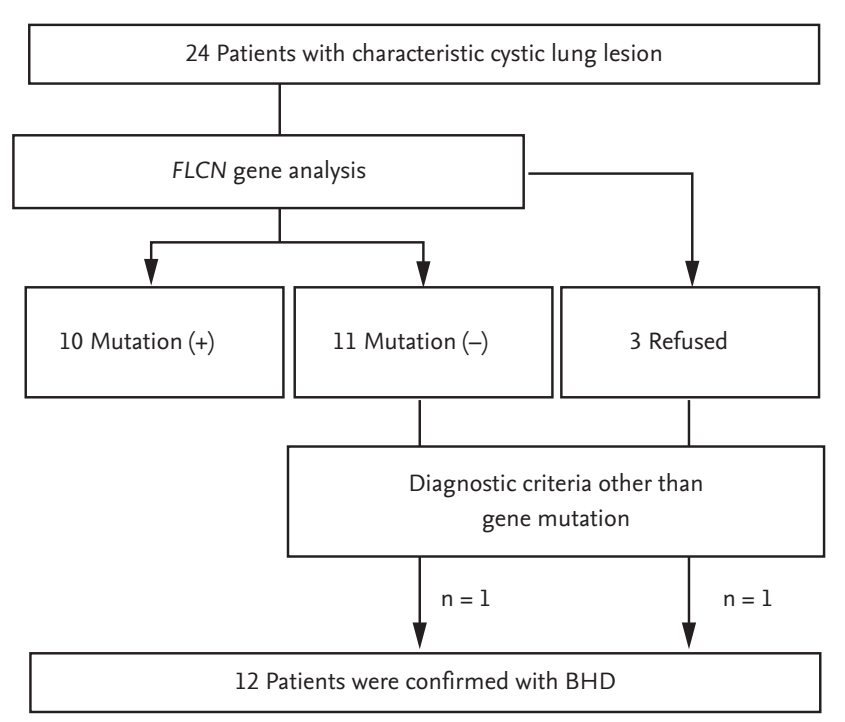

Figure 1. Flow diagram of patient recruitment. Twenty-four suspected patients with clinico-pathologic-radiologic features of Birt-Hogg-Dubé (BHD) syndrome were screened. According to the BHD diagnostic criteria, a total of 12 patients were diagnosed with BHD syndrome; 10 patients were confirmed by folliculin (FLCN) gene mutation analysis and the others by clinical diagnosis.

[4] performed a gene analysis of eight patients with lung cysts without skin and renal disease and found five patients had a FLCN germline mutation. Kunogi et al. [5] analyzed 36 patients with multiple lung cysts and found 25 patients with an FLCN mutation, among whom skin lesions were detected in $28 \%$ and typical skin lesion in only one (4\%). Furuya et al. [7] investigated 156 FLCN mutation carriers and 76 of them had skin papules; however, typical fibrofolliculomas were confirmed in six (3.8\%) only. In these studies, the authors suggested that pulmonary cysts, and often renal cancer, may be more informative than skin lesions in the diagnosis of BHD syndrome in Japanese patients. However, the clinicoradiologic features in Korean patients are not well defined. The purpose of this study was to define the clinicoradiologic features of Korean patients with BHD syndrome and to compare them with previous reports.

\section{METHODS}

\section{Study population}

Between January 2004 and November 2016, 24 suspected patients with clinico-pathologic-radiologic features of BHD syndrome were screened at Asan Medical Center, Seoul, South Korea. The diagnosis of BHD syndrome was based on the criteria proposed by the European BHD consortium [8]. The diagnosis was made when patients fulfilled at least one major or two minor criteria. The major criteria were as follows: (1) at least five fibrofolliculomas or trichodiscomas (at least one histologically confirmed, of adult onset); (2) pathogenic FLCN germline mutation. The minor criteria were as follows: (1) multiple lung cysts (bilateral basally located lung cysts with no other apparent cause, with or without spontaneous primary pneumothorax); (2) renal cancer (early onset before age 50 or multifocal or bilateral, or mixed chromophobe and oncocytic histology); (3) a first-degree relative with BHD [8]. Based on these criteria, 12 patients were diagnosed with BHD syndrome (10 confirmed by direct sequencing of the FLCN gene and two confirmed by clinical diagnosis) (Fig. 1). Other multiple cystic lung disorders, such as lymphangioleiomyomatosis (LAM), were excluded by characteristic high-resolution computed tomography (HRCT) and/or histologic findings. For example, the diffuse thin walled cysts with 2 to $5 \mathrm{~mm}$-sized, round or ovoid shape and surrounded by normal lung without regional sparing on HRCT images, and smooth-muscle cell proliferation in the pulmonary interstitium affecting vessels, airways, lymphatics, alveolar septa, and pleura on the histology supported the diagnosis of LAM [9].

The study was approved by the Institutional Review Board of Asan Medical Center (approval number: 20160744) and informed consent was waived because the data were analyzed anonymously and retrospectively.

\section{Clinical information}

Clinical and survival data for all patients were obtained from medical records, telephone interviews, and/or the records of the National Health Insurance of Korea. All clinical parameters and lung function data were obtained within 1 month after the initial visit.

Spirometry, total lung capacity by plethysmography, and diffusing capacity of the lung for carbon monoxide were measured according to the recommendation [10-12] and the results were expressed as percentages of normal predicted values. All patients underwent HRCT scans at their first visit. Abdominopelvic computed tomography (CT) or kidney ultrasonography was per- 
formed in patients who were first diagnosed with BHD to evaluate renal lesions. Patients who had skin lesions were referred to dermatologists and underwent punch biopsy.

\section{Radiologic assessments of HRCT images}

Chest CT images were reviewed by one chest radiologist (E.J.C.). The number, distribution, size and shape of pulmonary cysts were assessed on both axial and coronal reformatted images. The number of pulmonary cysts was classified into three groups: less than 50, 50 to 100, and more than 100. The distribution of pulmonary cysts was classified as upper dominant, lower dominant, or diffuse which was divided by the level of carina. The distribution was also classified as central dominant, peripheral dominant, or diffuse which was divided by the midline between hilar structure and costal pleural surface. The relation of the cysts to visceral pleura and interlobular fissures was also analyzed. Cyst size was measured along the long axis of the cysts. Cyst shape was classified as round, oval, lentiform, or irregular; round cysts were defined as having a smooth surface and a diameter ratio of long to short axes $\leq 1.1$, oval as having a diameter ratio of long to short axes $>1.1$, lentiform as being convex on both sides meeting at an acute angle, and all others as being irregular-shaped.

\section{Mutation analysis of the FLCN gene}

Genomic DNA was extracted from peripheral blood leukocytes. Eleven coding exons (exons 4 to 14) and exon-intron boundaries of the FLCN gene were amplified by polymerase chain reaction (PCR) using the primers designed by Primer3 (Supplementary Table 1) [13]. After PCR amplification, the PCR products were purified and directly sequenced using an $\mathrm{ABI}_{3130 x 1}$ Genetic Analyzer (Applied Biosystems, Foster City, CA, USA). DNA sequences were analyzed according to GenBank reference DNA sequences NG_0o8001.2 (genomic DNA), NM_144997.5 (mRNA), and NP_659434.2 (protein) (http:// www.ncbi.nlm.nih.gov).

\section{Statistical analysis}

All values were given as the mean \pm standard deviation for continuous variables or as percentages for categorical variables. Student's $t$ test or Mann-Whitney $U$ test was used for continuous data, and Pearson's chi-square test or Fisher exact test was used for categorical data. All statistical analyses were performed using SPSS version 21.0 (IBM Co., Armonk, NY, USA). All reported $p$ values are two-sided, and $p<0.05$ was considered as significant.

\section{RESULTS}

\section{Study population}

The median follow-up period was 52 months (range, 1 to 149 ). The mean age was 41.3 years, $66.7 \%$ were female, and $58.3 \%$ were ever-smokers (Table 1). Pneumothorax was the most common initial manifestation in seven patients $(58.3 \%)$, followed by asymptomatic screening (41.7\%).

A family of seven patients $(63.6 \%$, among total 11 non-family patients) had suspected clinical features of BHD syndrome (Table 1). Among total 12 patients, two (patient no. 2 and 9) were sisters; the older sister was diagnosed after the second event of pneumothorax and the younger sister underwent diagnostic evaluation by

Table 1. Baseline characteristics of the study population $(n=12)$

\begin{tabular}{lc}
\hline Characteristic & Value \\
\hline Age, yr & $41.3 \pm 12.0$ \\
Female sex & $8(66.7)$ \\
Smoking & \\
\hline Never-smokers & $5(41.7)$ \\
Ex-smokers & $3(25)$ \\
\hline Current-smokers & $4(33.3)$ \\
Smoking amount, pack-year & $8.2 \pm 3.3$ \\
\hline Body mass index, kg/m ${ }^{2}$ & $23.5 \pm 3.2$ \\
\hline Initial presentation & \\
\hline Pneumothorax & $7(58.3)$ \\
\hline Asymptomatic screening & $5(41.7)$ \\
\hline Family history & 7 \\
\hline Pneumothorax & $4(36.4)$ \\
\hline Pulmonary cyst & $2(18.2)$ \\
\hline Skin lesion & $2(18.2)$ \\
\hline Renal cancer & $1(9.1)$ \\
\hline Colon cancer & $4(36.4)$ \\
\hline
\end{tabular}

Values are presented as mean \pm SD or number (\%).

${ }^{a}$ Because two patients (patient no. 2 and 9) were sisters, the percentage was calculated for a total of 11 patients. 


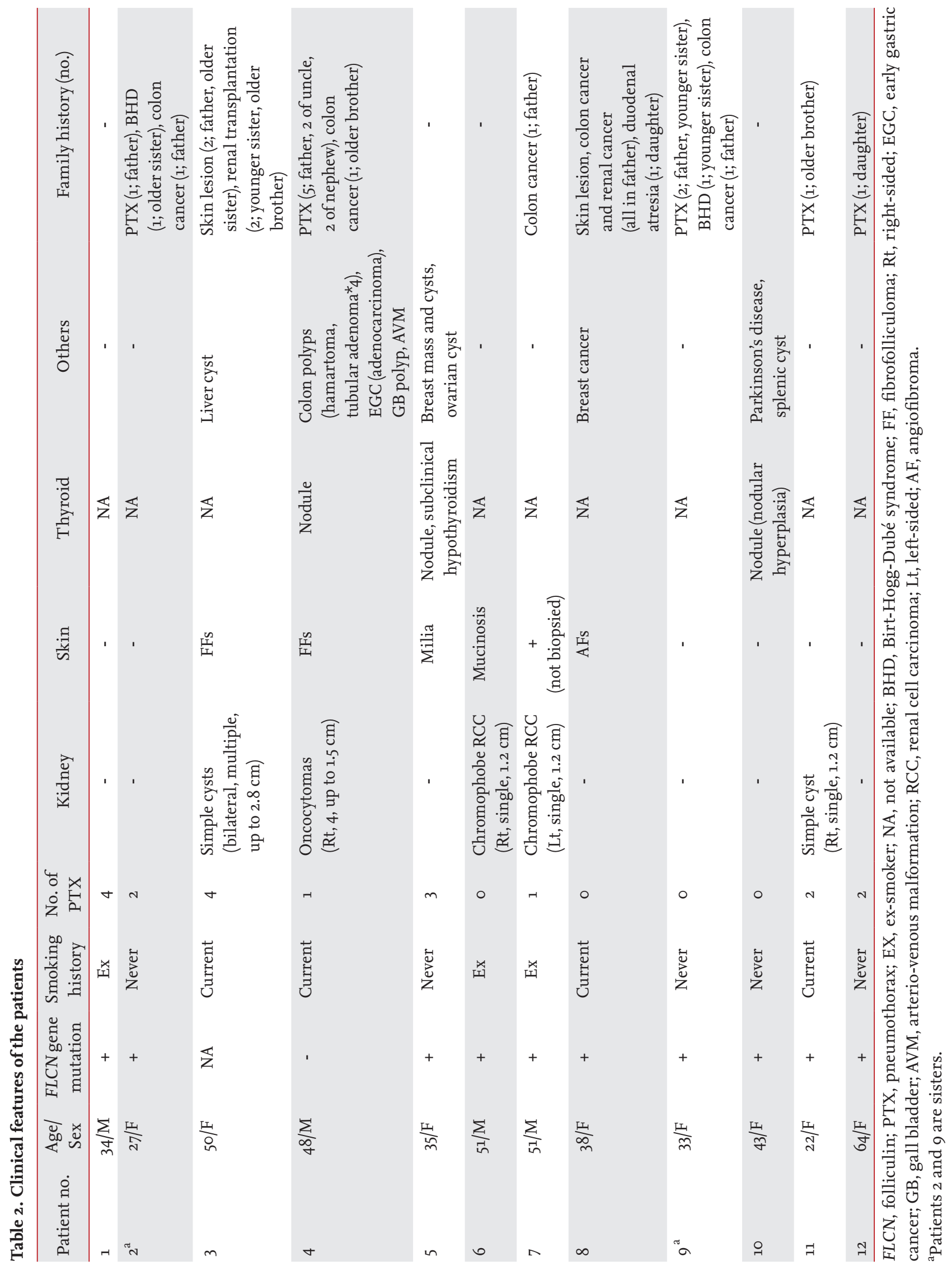


genetic counseling. Their other sister and father, not included in this study, also had histories of pneumothorax; however, they did not undergo diagnostic evaluation. The other patient (no. 4) had five family members with a history of pneumothorax. Four patients (36.4\%) had family histories of colon cancer.

Various comorbidities were found in these patients (Table 2). Thyroid nodules were detected in three patients (25\%), one of whom (no. 10) underwent a right thyroid lobectomy that revealed nodular hyperplasia. Extrapulmonary cystic lesions (liver, ovary, breast, and spleen) were also found in three patients (25\%), and arteriovenous malformation in brain, early gastric cancer, colon polyps (tubular adenoma and hamartoma), and a gall bladder polyp were found in one (no. 4).

\section{Mutation analysis of the $F L C N$ gene}

Five different mutations of the FLCN gene were confirmed by genetic testing in 10 patients (Table 3). The most frequent mutation (40\%) was the duplication of cytosine in the C8 tract of exon 11 (c.1285dupC), as was reported in previous studies [2,14-17]. One patient (no. 8) harbored a novel heterozygous sequence variant of c.31T>C (p.C11R) in exon 4 that replaces a cysteine with an arginine at codon 11.This mutation was not found in the gnomAD browser (http://gnomad.broadinstitute. org/) and was predicted to be probably damaged by PolyPhen-2 (http://genetics.bwh.harvard.edu/pph2/).

\section{Pulmonary manifestation}

All 12 patients had multiple pulmonary cysts (Table 4). The total number of pulmonary cysts varied, and a number of 50 to 100 cysts per patient was the most common (50\%). Cyst size varied from 3 to $70 \mathrm{~mm}$. The most dominant cyst shape was irregular in 10 patients (83.3\%), followed by lentiform $(n=1,8.3 \%)$ and round $(\mathrm{n}=1,8.3 \%)$, respectively. The cysts were distributed in the predominantly lower and peripheral regions of the lungs (Fig. 2). Most of the patients had fissural (91.7\%) and subpleural (100\%) cysts. Pneumothorax developed in eight patients (66.7\%) and $75 \%$ were recurrent (up to five times during follow-up). The mean age of the initial episodes of pneumothorax was $29.8 \pm 8.4$ years. The number, maximal size, and distribution of cysts, along with patient age and smoking status were not associated with pneumothorax.
Most of the patients had normal lung function (58.3\%); however, mild restrictive pattern was also detected in 33.3\% (Table 5). Bronchoalveolar lavage (BAL) was performed in two patients, and BAL fluid analysis showed nonspecific results. Wedge resection was performed in four patients due to pneumothorax. The specimen showed multiple small intraparenchymal cysts rimmed by thin, fibrous walls or normal pulmonary parenchyma (Fig. 2).

\section{Renal manifestation}

Simple cysts were detected in two patients (16.7\%) and renal cancers in three (25\%), two of whom carried an FLCN mutation in exon 11. The mean age at the initial diagnosis of renal cancer was $54 \pm 4$ years. All three patients underwent partial nephrectomy. The histologic type was chromophobe in two patients (no. 6 and 7) and oncocytic in one (no. 4). Patient no. 4 had a $0.9-\mathrm{cm}$ renal nodule that was difficult to differentiate at the time of diagnosis. Ten years later, the size of the nodule had increased from 0.9 to $1.2 \mathrm{~cm}$, and oncocytoma was confirmed by surgery. In patient no. 6, a $1.2 \mathrm{~cm}$-sized renal cancer developed 4 years after the diagnosis.

\section{Skin manifestation}

Skin lesions were detected in six patients. Most of the lesions presented as small, dome-shaped, whitish papules visualized on the face, neck, and/or upper trunk. Skin biopsies were performed in five patients. Fibrofolliculoma was confirmed in two patients (16.7\%), angiofibroma in one (8.3\%), and mucinosis and milia, which do not commonly present in BHD syndrome, in two (16.7\%).

\section{Clinical course and outcome}

During follow-up, lung function remained almost unchanged (Fig. 3). Pneumothorax recurred in two patients (16.7\%; no. 1 and 2). One patient (no. 1) had repeated recurrence of pneumothorax even though he had undergone bullectomy twice. Another patient (no. 2) had already had a history of pneumothorax twice; however, he experienced three more pneumothoraces during follow-up. One patient (no. 8) developed breast cancer 3 years after the diagnosis of BHD syndrome and underwent breast conserving surgery, followed by adjuvant chemotherapy and radiotherapy. No one died during the follow-up. 
Table 3. Mutations of the FLCN gene detected in the patients

\begin{tabular}{lcccc}
\hline Location & Nucleotide change & Protein change & Patient no. \\
\hline Exon 4 & c.31T>C & p.C11R & 8 \\
Exon 6 & c.469-471del(TTC) & p.F157del & 1,11 & $5,6,7,10$ \\
Exon 11 & c.1285dupC & p.H429Pfs 27 & 12 \\
Exon 12 & c.1429C $>$ T & p.R $477^{*}$ & p.F519Lfs* 18 & 2,9 \\
Exon 14 & c.1557del & & \\
\hline
\end{tabular}

An asterisk (*) stands for a stop codon for amino acids.

Table 4. Comparison of clinicoradiologic features between patients with and without a history of pneumothorax

\begin{tabular}{|c|c|c|c|c|}
\hline \multirow{2}{*}{ Characteristic } & \multirow{2}{*}{ All $(\mathrm{n}=12)$} & \multicolumn{2}{|c|}{ Pneumothorax } & \multirow{2}{*}{$p$ value } \\
\hline & & Yes $(\mathrm{n}=8)$ & No $(n=4)$ & \\
\hline Age & $41.3 \pm 12.0$ & $41.4 \pm 14.1$ & $41.3 \pm 7.7$ & 0.987 \\
\hline Female sex & $8(66.7)$ & $5(62.5)$ & $3(75)$ & $>0.999$ \\
\hline Ever-smoker & $7(58.3)$ & $5(62.5)$ & $2(50)$ & $>0.999$ \\
\hline No. of pulmonary cysts & & & & $0.547^{\mathrm{a}}$ \\
\hline Less than 50 & $4(33 \cdot 3)$ & $2(25)$ & $2(50)$ & \\
\hline $50-100$ & $6(50)$ & $4(50)$ & $2(50)$ & \\
\hline More than 100 & $2(16.7)$ & $2(25)$ & $\mathrm{O}$ & \\
\hline \multicolumn{5}{|l|}{ Size of pulmonary cysts } \\
\hline Minimum, mm & $4.4 \pm 1.3$ & $4.3 \pm 1.2$ & $4.8 \pm 1.7$ & 0.559 \\
\hline Maximum, mm & $35.1 \pm 16.9$ & $30.9 \pm 13.9$ & $43 \cdot 5 \pm 21.3$ & 0.239 \\
\hline \multicolumn{2}{|c|}{ Most predominant shape of pulmonary cysts } & & & $>0.999^{b}$ \\
\hline Round & $1(8.3)$ & $1(12.5)$ & o & \\
\hline Oval & o & o & o & \\
\hline Lentiform & $1(8.3)$ & o & $1(25)$ & \\
\hline Irregular & $10(83 \cdot 3)$ & $7(87.5)$ & $3(75)$ & \\
\hline \multicolumn{2}{|c|}{ Distribution of pulmonary cysts (upper/lower) } & & & $>0.999$ \\
\hline Upper & o & o & O & \\
\hline Lower & $10(83 \cdot 3)$ & $7(87 \cdot 5)$ & $3(75)$ & \\
\hline Diffuse & $2(16.7)$ & $1(12.5)$ & $1(25)$ & \\
\hline \multicolumn{2}{|c|}{ Distribution of pulmonary cysts (central/peripheral) } & & & $>0.999$ \\
\hline Central & o & O & O & \\
\hline Peripheral & $8(67.7)$ & $5(62.5)$ & $3(75)$ & \\
\hline Diffuse & $4(33 \cdot 3)$ & $3(37.5)$ & $1(25)$ & \\
\hline
\end{tabular}

Values are presented as mean \pm SD or number (\%).

${ }^{a}$ The group of cyst number was reclassified into two groups (less than 50 or 50 or more) when compared to the presence or absence of pneumothorax due to the small number of patients.

${ }^{\mathrm{b}}$ The group of predominant cyst shape was reclassified into two groups (irregular or not-irregular) when compared to the presence or absence of pneumothorax due to the small number of patients. 


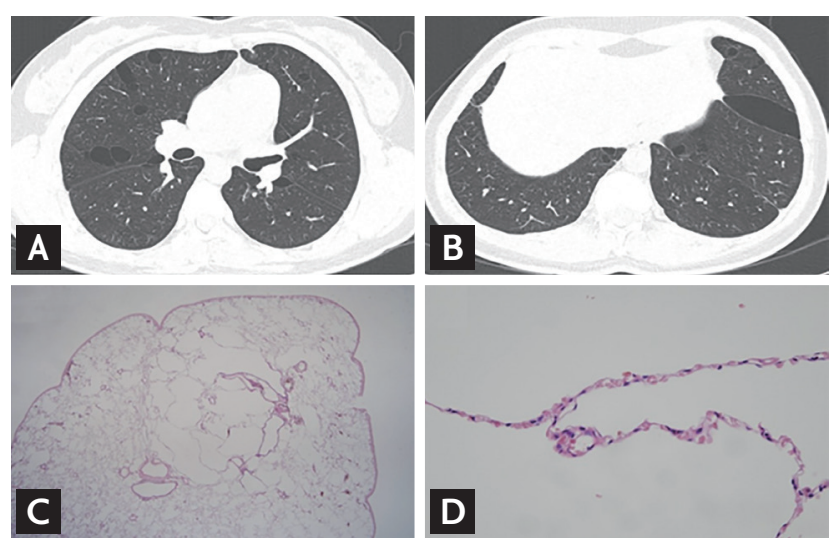

Figure 2. Examples of chest computed tomography and histologic findings of the study patients. The pulmonary cysts in Birt-Hogg-Dubé (BHD) are distributed in predominantly lower, peripheral and subpleural regions of the lungs (A: upper lobes; B: lower lobes). Lung specimens present multiple small intraparenchymal cysts rimmed by thin fibrous walls or normal pulmonary parenchyma. (C: ×40; D: ×400).

Table 5. Baseline physiologic parameters and bronchoalveolar lavage fluid findings of the subjects $(n=12)$

\begin{tabular}{lc}
\hline Characteristic & Value \\
\hline Pulmonary function test, \% predicted & \\
\hline FVC & $81.8 \pm 13.1$ \\
$\mathrm{FEV}_{1}$ & $79.9 \pm 13.6$ \\
$\mathrm{FEV}_{1} / \mathrm{FVC}$ & $0.8 \pm 0.0$ \\
\hline DLco & $83.6 \pm 19.5$ \\
\hline TLC & $86.7 \pm 10.7$ \\
\hline Pulmonary function test pattern & $7(58.3)$ \\
\hline Normal & $1(8.3)$ \\
\hline Obstructive pattern & $4(33.3)$ \\
\hline Restrictive pattern & \\
\hline 6-Minute walk test & $489.6 \pm 68.9$ \\
\hline Distance, meter & $98.3 \pm 1.5$ \\
\hline Initial SpO,$\%$ & $96.4 \pm 1.9$ \\
\hline Lowest SpO & \\
\hline Bronchoalveolar lavage & 2 \\
\hline WBC, cells/uL & $195.0 \pm 7.1$ \\
\hline Neutrophils, \% & $3.0 \pm 1.4$ \\
\hline Lymphocytes, \% & $11.0 \pm 1.4$ \\
\hline Values are presented as & \\
\hline
\end{tabular}

Values are presented as mean $\pm \mathrm{SD}$ or number (\%).

FVC, forced vital capacity; $\mathrm{FEV}_{1}$, forced expiratory volume in 1 second; DLco, diffusing capacity of the lung for carbon monoxide; TLC, total lung capacity; $\mathrm{SpO} 2$, peripheral oxygen saturation; WBC, white blood cell.

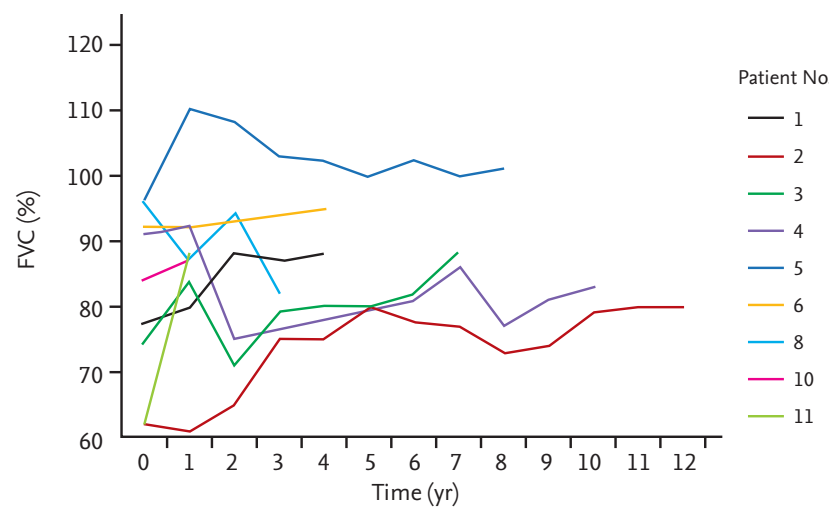

Figure 3. Changes in forced vital capacity (FVC) during follow-up. Nine of 12 patients underwent regular pulmonary function test follow-up. Lung function remained almost unchanged during follow-up.

\section{DISCUSSION}

The clinicoradiologic features of BHD syndrome in Korean patients were similar to previous reports. All patients had multiple variable-sized pulmonary cysts that were commonly irregular-shaped and distributed in the lower and peripheral regions of the lungs. Pneumothorax was the most common presenting feature (66.7\%), and the duplication of cytosine in the C8 tract of exon 11 (c.1285dupC) was the most common mutation (40\%). However, in our cohort, skin lesions were less frequent (25.0\%) compared to previous reports, and a novel mutation of c.31T>C in exon 4 was found.

The FLCN gene is located on chromosome 17p11.2 and is comprised of 14 exons with the transcription start site in exon 4. Although mutations can occur anywhere in the FLCN gene, a frameshift caused by insertions or deletions in the cytosine 8 nucleotide of exon 11 was the most common (50\%) mutation in a previous study [14]. The detection rate of an FLCN gene mutation was approximately $84 \%$ to $88 \%$ in BHD syndrome [14,15], and 69.4\% in cystic lung disease of undetermined causes [5]. In this study, an FLCN gene mutation was detected in 10 of 21 suspected patients (47.6\%) and 10 of 11 (90.9\%) confirmed cases with BHD syndrome who underwent genetic analysis. In addition, a novel mutation was found in this study: a heterozygous sequence variant of c.31 T>C (p.C11R) in exon 4 replacing a cysteine with an arginine at codon 11. This mutation was predicted to be deleterious by in silico prediction program, PolyPhen-2. 
This patient (no. 8) presented with multiple lung cysts distributed mainly in the lower lobe and angiofibroma, corresponding with the known clinical features of BHD syndrome.

In previous reports, BHD syndrome presented pulmonary cysts in $79 \%$ to $100 \%$ and pneumothorax in $24 \%$ to $35 \%$, which occurred repeatedly in $75 \%$ to $86.2 \%$ [5,14,15,18-20]. However, our study showed more frequent pneumothorax than in previous studies of whites $[8,15,18,20,21]$ : pulmonary cysts were detected in all subjects (100\%) and pneumothorax in $66.7 \%$, which recurred in $75 \%$. Other previous reports supported our findings $[5,7,22,23]$. Park et al. [22] reported that 50\% of the Korean subjects had a history of pneumothorax. Some Japanese studies also reported that $73.7 \%$ to $96.7 \%$ of patients experienced pneumothorax [5,7,23]. This suggests that Asian patients with BHD syndrome may have a higher risk of pneumothoroax than white patients.

Despite the multiple pulmonary cysts, most patients had normal lung function, and this might be attributed to the histopathologic findings of the lung parenchyma, which generally appeared normal [24]. Lung function remained almost unchanged during follow-up, and this is distinctive from other cystic lung diseases such as LAM [23]. In previous reports, maximal diameter and volume, total volume and number of cysts, and age were significantly associated with pneumothorax [20,25]; however, we could not find any correlation between these findings and pneumothorax, probably due to the small number.

Renal cancers in patients with BHD syndrome are known to be diagnosed at about 50 years old $[15,26]$, and show bilateral or multifocal lesions in more than half of patients $[25,27]$. In this study, the age of diagnosis was similar to previous results; however, renal cancers were presented as single and unilateral mass. Chromophobe was the most common histologic type, followed by oncocytoma, similar to previous studies [28], and there were no cases of metastasis even though one existed for 10 years. A few cases of metastatic renal caner have been reported in BHD syndrome to date, all of which revealed the histology of clear-cell, hybrid clear-cell/papillary or hybrid clear-cell/chromophobe type [15,19,26,29,30]. This suggests that oncocytoma or chromophobe renal cancers may have an indolent course and be less likely to metastasize as seen in this study $[8,24]$.

Skin lesions are the most common feature of BHD syndrome (up to 80\%) [31], usually appearing after the age of 20 as multiple dome-shaped whitish papules on the face, mainly on the nose and cheeks [8]. Histologically, these consist of fibrofollicullomas, trichodiscomas, and angiofibromas. However, skin lesions were only detected in three patients (25\%) in this study (two fibrofolliculomas and one angiofibroma). Some studies in Japanese patients also reported that approximately $20 \%$ to $29 \%$ of patients had cutaneous manifestations [5-7]. Park et al. [22] also reported six Korean patients with BHD, none of whom had typical renal or skin lesions. Murakami et al. [6] suggested that the smaller total hair count and number of follicular units in Asians than whites may account for the few unremarkable fibrofolliculomas. Fibrofolliculoma, or benign hair follicle tumor, was the most common type in this study, followed by angiofibroma and mucinosis. Although angiofibroma is usually associated with tuberous sclerosis complex, it has also been reported in BHD syndrome [8,15,32]. Mucin-containing skin lesions were reported in previous studies; however, none of them have proved to be related with BHD syndrome [1,27,33-37].

In our study, cysts were also detected in various extrapulmonary organs (kidney, liver, ovary, breast, and spleen). A previous study supported our findings; Furuya et al. [7] observed renal (19.2\%) and liver cysts (9.6\%) in patients with an FLCN gene mutation and they suggested a relationship between FLCN gene mutation and the cysts in these organs. Thyroid nodules were present in $25 \%$ of the patients, one of whom showed nodular hyperplasia. Kluger et al. [18] reported high prevalence (65\%) of thyroid nodules in BHD syndrome by ultrasound screening. Several cases of multi-nodular goiter [38,39], hypothyroidism [33], Hashimoto's thyroiditis [40], and thyroid cancer $[15,41]$ were also reported in BHD syndrome.

Early gastric cancer and colon polyps (tubular adenoma and hamartoma) were detected in one patient at the same time, and five (41.7\%) had a family history of colon cancer. Colon polyps or adenocarcinoma had previously been reported to be related to BHD syndrome [34,42]; however, a large cohort study by Zbar et al. [25] showed no significant increase of colon polyps in patients with BHD syndrome compared to those without. Some studies also suggested that an increased risk of colorectal cancer might apply only to specific subgroups of pa- 
tients $[16,40]$. It is possible that specific FLCN genotypes or genetic alterations in other genes may increase the risk for colonic neoplasia in an individual affected with BHD syndrome.

This study has some limitations. First, this was a retrospective study conducted in a single, tertiary referral center. However, the clinicoradiologic features of patients with BHD syndrome in our study were comparable to those in previous studies. Second, the study population was small, but it included the largest number of South Korean patients compared to previous studies. Third, mutation of the FLCN gene was not confirmed in two of 12 patients (no. 3 refused gene analysis and no. 4 did not have a mutated FLCN gene); however, they satisfied the criteria suggested by the BHD consortium and showed compatible clinicoradiologic features of BHD syndrome.

In conclusion, although Korean patients with BHD syndrome share clinicoradiologic features reported in previous studies, they also show some different characteristics, such as a higher risk of pneumothorax, less frequent skin lesions and a novel mutation of c.31T $>C$ in exon 4. These findings need to be confirmed in a larger population.

\section{KEY MESSAGE}

1. Korean patients with Birt-Hogg-Dubé syndrome may have a higher risk of pneumothorax, less frequent skin lesions, and a novel FLCN mutation compared to previous reports.

2. Multiple bilateral and basal-predominant cysts were the most common radiologic features.

3. Lung function remained almost unchanged during follow-up, but some patients had repeated recurrence of pneumothorax.

\section{Conflict of interest}

No potential conflict of interest relevant to this article was reported.

\section{Acknowledgments}

This study was supported by a grant of Basic Science Research Program through the National Research Foundation of Korea (NRF) funded by the Ministry of Science,
ICT and Future Planning (NRF-2016R1A2B4016318). We thank Ms. Vanessa Topping from the Scientific Publications Team at Asan Medical Center for her editorial assistance in preparing this manuscript.

\section{REFERENCES}

1. Birt AR, Hogg GR, Dube WJ. Hereditary multiple fibrofolliculomas with trichodiscomas and acrochordons. Arch Dermatol 1977;113:1674-1677.

2. Nickerson ML, Warren MB, Toro JR, et al. Mutations in a novel gene lead to kidney tumors, lung wall defects, and benign tumors of the hair follicle in patients with the Birt-Hogg-Dubé syndrome. Cancer Cell 2002;2:157-164.

3. Hartman TR, Nicolas E, Klein-Szanto A, et al. The role of the Birt-Hogg-Dubé protein in mTOR activation and renal tumorigenesis. Oncogene 2009;28:1594-1604.

4. Gunji Y, Akiyoshi T, Sato T, et al. Mutations of the Birt Hogg Dube gene in patients with multiple lung cysts and recurrent pneumothorax. J Med Genet 2007;44:588-593.

5. Kunogi M, Kurihara M, Ikegami TS, et al. Clinical and genetic spectrum of Birt-Hogg-Dube syndrome patients in whom pneumothorax and/or multiple lung cysts are the presenting feature. J Med Genet 2010;47:281-287.

6. Murakami Y, Wataya-Kaneda M, Tanaka M, et al. Two Japanese cases of birt-hogg-dubé syndrome with pulmonary cysts, fibrofolliculomas, and renal cell carcinomas. Case Rep Dermatol 2014;6:20-28.

7. Furuya M, Yao M, Tanaka R, et al. Genetic, epidemiologic and clinicopathologic studies of Japanese Asian patients with Birt-Hogg-Dubé syndrome. Clin Genet 2016;90:403412.

8. Menko FH, van Steensel MA, Giraud S, et al. Birt-HoggDubé syndrome: diagnosis and management. Lancet Oncol 2009;10:1199-1206.

9. Seaman DM, Meyer CA, Gilman MD, McCormack FX. Diffuse cystic lung disease at high-resolution CT. AJR Am J Roentgenol 2011;196:1305-1311.

10. Miller MR, Hankinson J, Brusasco V, et al. Standardisation of the single-breath determination of carbon monoMacintyre N, Crapo RO, Viegi G, et al. Standardisation of the single-breath determination of carbon monoxide uptake in the lung. Eur Respir J 2005;26:720-735.

11. Macintyre N, Crapo RO, Viegi G, et al. Standardisation of the single-breath determination of carbon monoxide up- 
take in the lung. Eur Respir J 2005;26:720-735.

12. Wanger J, Clausen JL, Coates A, et al. Standardisation of the measurement of lung volumes. Eur Respir J 2005;26:511-522.

13. Untergasser A, Cutcutache I, Koressaar T, et al. Primer3: new capabilities and interfaces. Nucleic Acids Res 2012;40:e115.

14. Schmidt LS, Nickerson ML, Warren MB, et al. Germline BHD-mutation spectrum and phenotype analysis of a large cohort of families with Birt-Hogg-Dubé syndrome. Am J Hum Genet 2005;76:1023-1033.

15. Toro JR, Wei MH, Glenn GM, et al. BHD mutations, clinical and molecular genetic investigations of Birt-HoggDubé syndrome: a new series of 50 families and a review of published reports. J Med Genet 2008;45:321-331.

16. Nahorski MS, Lim DH, Martin L, et al. Investigation of the Birt-Hogg-Dube tumour suppressor gene (FLCN) in familial and sporadic colorectal cancer. J Med Genet 2010;47:385-390.

17. Khoo SK, Bradley M, Wong FK, Hedblad MA, Nordenskjold M, Teh BT. Birt-Hogg-Dubé syndrome: mapping of a novel hereditary neoplasia gene to chromosome 17p12-q11.2. Oncogene 2001;20:5239-5242.

18. Kluger N, Giraud S, Coupier I, et al. Birt-Hogg-Dubé syndrome: clinical and genetic studies of 10 French families. Br J Dermatol 2010;162:527-537.

19. Houweling AC, Gijezen LM, Jonker MA, et al. Renal cancer and pneumothorax risk in Birt-Hogg-Dubé syndrome; an analysis of 115 FLCN mutation carriers from 35 BHD families. Br J Cancer 2011;105:1912-1919.

20. Toro JR, Pautler SE, Stewart L, et al. Lung cysts, spontaneous pneumothorax, and genetic associations in 89 families with Birt-Hogg-Dubé syndrome. Am J Respir Crit Care Med 2007;175:1044-1053.

21. Gupta N, Sunwoo BY, Kotloff RM. Birt-Hogg-Dubé syndrome. Clin Chest Med 2016;37:475-486.

22. Park HJ, Park CH, Lee SE, et al. Birt-Hogg-Dube syndrome prospectively detected by review of chest computed tomography scans. PLoS One 2017;12:e0170713.

23. Tobino K, Hirai T, Johkoh T, et al. Differentiation between Birt-Hogg-Dubé syndrome and lymphangioleiomyomatosis: quantitative analysis of pulmonary cysts on computed tomography of the chest in 66 females. Eur J Radiol 2012;81:1340-1346.

24. Dal Sasso AA, Belem LC, Zanetti G, et al. Birt-Hogg-Dubé syndrome. State-of-the-art review with emphasis on pul- monary involvement. Respir Med 2015;109:289-296.

25. Zbar B, Alvord WG, Glenn G, et al. Risk of renal and colonic neoplasms and spontaneous pneumothorax in the Birt-Hogg-Dubé syndrome. Cancer Epidemiol Biomarkers Prev 2002;11:393-400.

26. Pavlovich CP, Grubb RL 3rd, Hurley K, et al. Evaluation and management of renal tumors in the Birt-Hogg-Dubé syndrome. J Urol 2005;173:1482-1486.

27. Toro JR, Glenn G, Duray P, et al. Birt-Hogg-Dubé syndrome: a novel marker of kidney neoplasia. Arch Dermatol 1999;135:1195-1202.

28. Pavlovich CP, Walther MM, Eyler RA, et al. Renal tumors in the Birt-Hogg-Dubé syndrome. Am J Surg Pathol 2002;26:1542-1552.

29. Claessens T, Weppler SA, van Geel M, et al. Neuroendocrine carcinoma in a patient with Birt-Hogg-Dubé syndrome. Nat Rev Urol 2010;7:583-587.

30. Nakamura M, Yao M, Sano F, et al. A case of metastatic renal cell carcinoma associated with Birt-Hogg-Dubé syndrome treated with molecular-targeting agents. Hinyokika Kiyo 2013;59:503-506.

31. Aivaz O, Berkman S, Middelton L, Linehan WM, DiGiovanna JJ, Cowen EW. Comedonal and cystic fibrofolliculomas in Birt-Hogg-Dube syndrome. JAMA Dermatol 2015;151:770-774.

32. Schaffer JV, Gohara MA, McNiff JM, Aasi SZ, Dvoretzky I. Multiple facial angiofibromas: a cutaneous manifestation of Birt-Hogg-Dubé syndrome. J Am Acad Dermatol 2005:53(2 Suppl 1):S108-S111.

33. Nadershahi NA, Wescott WB, Egbert B. Birt-Hogg-Dubé syndrome: a review and presentation of the first case with oral lesions. Oral Surg Oral Med Oral Pathol Oral Radiol Endod 1997;83:496-500.

34. Rongioletti F, Hazini R, Gianotti G, Rebora A. Fibrofolliculomas, tricodiscomas and acrochordons (Birt-HoggDubé) associated with intestinal polyposis. Clin Exp Dermatol 1989;14:72-74.

35. Chung JY, Ramos-Caro FA, Beers B, Ford MJ, Flowers F. Multiple lipomas, angiolipomas, and parathyroid adenomas in a patient with Birt-Hogg-Dube syndrome. Int J Dermatol 1996;35:365-367.

36. Scalvenzi M, Argenziano G, Sammarco E, Delfino M. Hereditary multiple fibrofolliculomas, trichodiscomas and acrochordons: syndrome of Birt-Hogg-Dubè. J Eur Acad Dermatol Venereol 1998;11:45-47.

37. Lindor NM, Hand J, Burch PA, Gibson LE. Birt-Hogg- 
Dube syndrome: an autosomal dominant disorder with predisposition to cancers of the kidney, fibrofolliculomas, and focal cutaneous mucinosis. Int J Dermatol 2001;40:653-656.

38. Welsch MJ, Krunic A, Medenica MM. Birt-Hogg-Dubé syndrome. Int J Dermatol 2005;44:668-673.

39. Drummond C, Grigoris I, Dutta B. Birt-Hogg-Dubé syndrome and multinodular goitre. Australas J Dermatol 2002;43:301-304.

40. Khoo SK, Giraud S, Kahnoski K, et al. Clinical and ge- netic studies of Birt-Hogg-Dubé syndrome. J Med Genet 2002;39:906-912.

41. Fahmy W, Safwat AS, Bissada NK, et al. Multiple/bilateral renal tumors in patients with Birt-Hogg-Dubé syndrome. Int Urol Nephrol 2007;39:995-999.

42. Le Guyadec T, Dufau JP, Poulain JF, Vaylet F, Grossin M, Lanternier G. Multiple trichodiscomas associated with colonic polyposis. Ann Dermatol Venereol 1998;125:717719. 
Lee JH, et al. Birt-Hogg-Dubé syndrome in Korean

Supplementary Table 1. Primers used for polymerase chain reaction amplification

\begin{tabular}{lll}
\hline & \multicolumn{1}{c}{ Forward primer } & Reverse primer \\
\hline Exon 4 & cctggggaggttcatggagt & acagtcaggatgagcggaaa \\
Exon 5 & ctttccgagctcagattgc & tgtaccctgtgctgtgctg \\
Exon 6 & cttaacgctggctgatttgtg & gtgcactggctgtaagcaga \\
Exon 7 & cgattggactggtggcacttt & ggactgttctcccaaatcca \\
Exon 8 & ctcgttgacttgtggaactgc & agcccacctgtgaattt \\
Exon 9 & cgcagccaggaatctacact & tatggctaataccccaacg \\
Exon 10 & ctgagcctgtctttgctct & agtggagaccgtgtggtg \\
Exon 11 & cactgtgggctgagagtctg & tccacaacccatgacagaga \\
Exon $12-13$ & ccactgacctgggatgagc & agctcctctttggaaacagc \\
Exon 14 & ctcctgctggtgccaaag & ctgatggtttccttcctt \\
\hline
\end{tabular}

Primers were designed with Primer3 [13] using sequences from GenBank (Accession number NT_010718.16). 